\title{
"Go In With An Open Mind": Perceptions Of Exoskeleton Use During Gait Rehabilitation In An Acute Hospital Setting Following Stroke.
}

Caitlin McDonald ( $\sim$ caitlin.mcdonald@ucdconnect.ie)

University College Dublin

\section{Caitriona Fingleton}

Mater Misericordiae University Hospital

\section{Sean Murphy}

Mater Misericordiae University Hospital

Olive Lennon

University College Dublin

\section{Research Article}

Keywords: Neurological rehabilitation, stroke, biomedical engineering, robotic assisted gait training, exoskeleton, physiotherapy, gait rehabilitation, qualitative research

Posted Date: March 8th, 2022

DOI: https://doi.org/10.21203/rs.3.rs-1325223/v2

License: (c) (i) This work is licensed under a Creative Commons Attribution 4.0 International License. Read Full License 


\section{Abstract}

Background: Robotic gait devices allow intensive high repetition of the gait cycle in individuals with locomotor disability, with reduced therapist effort. The addition of robotic-assisted gait training (RAGT) to usual rehabilitation post-stroke improves the likelihood of regaining independent walking, with maximum efficacy identified in the acute and subacute phases of stroke. The study explores the usability and acceptance of RAGT among persons with stroke in an acute hospital setting and examines users' perceptions of two different modes of robotic assistance provided during rehabilitation.

Methods: A mixed-methods approach comprised semi-structed interviews of end-user perspectives of RAGT in an acute hospital setting following stroke and two 10-point Likert scales rating how comfortable and how natural robotic gait felt using different assistance modes. Content analysis of qualitative data was undertaken with results synthesised by common meaning units. Quantitative data were reported using summary statistics, with Spearmann's correlation co-efficient examining the relationship between Likert scale ratings and measures of participants' stroke related disability.

Results: Ten individuals ( 6 men; 4 women; mean age of $64.5 . \pm 13$ years) were recruited from an acute hospital following admission with a stroke diagnosis. Content analysis of interviews identified discussion units centring around positive aspects of the device related to how helpful the device was, negative aspects related to set-up time, weight of the device and multiple instructions during use. Initially participants identified that the device could look intimidating and that they feared falling in the device but subsequently identified the correct mindset for using the device is to trust the technology and not be afraid. Mean ratings of device comfort $(7.94 \pm 1.4)$ and how natural walking felt $(7.05 \pm 1.9)$ were favourable. Interestingly, a strong relationship was identified, whereby the higher the level of disability, the more natural participants rated walking in the device in maximal assistance mode (rho $=0.62$ ).

Conclusions: This study suggests individuals in the early phases of stroke perceive RAGT to be acceptable and helpful in the main, with some associated negative aspects. The device was rated as comfortable and natural to use and those with greater disability rate the assisted walking was more natural.

\section{Introduction}

Stroke is a leading cause of mortality and morbidity worldwide. In 2019, over 6.5 million deaths and 143 million disability-adjusted life-years (DALYs) resulted from stroke globally (1). Hemiplegia is one of the most common impairments after stroke and significantly reduces locomotor performance $(2,3)$. Although the majority of stroke survivors achieve independent gait, many do not reach a walking level that enables them to perform all activities of daily living (4). For example, only $7 \%$ of patients discharged from rehabilitation following stroke reported meeting the criteria for community walking (5). At six months post-stroke $11 \%$ of survivors are unable to ambulate, with only $14 \%$ recovering independent gait with no walking aids (6). Individuals following stroke commonly demonstrate an altered gait pattern, (7) 
in compensation for impairments such as neuromuscular weakness, altered muscle tone, poor motor control, and soft tissue contractures. This results in increased energy expenditure during overground walking when compared to healthy individuals (8). Gait recovery is a major rehabilitation objective poststroke (9), with stroke survivors and other relevant stakeholders identifying gait rehabilitation and specifically electromechanically assisted gait training as a main priority for future research (10). Effective rehabilitation for this population requires high intensity, repetitive, and task-specific training (11), with intensity of practice strongly associated with functional gait outcomes (12).

Exoskeleton or robotic gait devices enable people to walk with electromechanical assistance to achieve a more normal gait pattern and can allow intensive high repetition of the gait cycle with reduced therapist involvement (13). The addition of robotic-assisted gait training (RAGT) to usual post-stroke rehabilitation has been shown to improve the likelihood of regaining independent walking following a stroke (14). While combining exoskeleton-based gait training with conventional physiotherapy can promote improved mobility and favourable neuroplastic changes in patients with chronic stroke (15), it is in the first three months after stroke (acute and subacute stages) when robotic gait training has shown most efficacy by meta-analysis (14). This is in keeping with data that supports the majority of neuroplastic changes as occurring in the subacute stage (16), during which the brain's response to injury is heightened and rehabilitation may be more effective (17). The best achievable neurological outcome is usually reached (in $80 \%$ of all patients) within 5 weeks, with $95 \%$ reaching their best outcome of functional ability at 3 months after stroke onset $(18,19)$.

Despite evidence of efficacy for RAGT in conjunction with routine rehabilitation in the earlier phases after stroke in gait restoration, limited data provide insights into stroke survivors', the primary end-users, perceptions of these devices in the acute stage. A qualitative meta-synthesis of stakeholders' perceptions of robotics in motor rehabilitation reports robotics to be generally acceptable, useful and beneficial (physically, psychologically, and socially) in rehabilitation (20). However, the review included only one study specifically focussed on gait rehabilitation after stroke (21), including only individuals in the chronic phases of stroke. Since this review, two further qualitative studies were identified reporting perspectives on RAGT in the chronic stage of stroke (> 12 weeks) (22) and in the subacute phase following transfer to a rehabilitation centre (23). In these later stages, exoskeleton interventions are generally well-received by physical therapists and stroke survivors with expressed excitement and hopefulness about exoskeleton technology. However, disappointment was registered by some individuals where the technology did not yet meet their expectations. A more adaptive device that augmented their natural gait as opposed to one that imposed a pre-set gait pattern on them was desired. One of the studies, addressing robotic-assisted gait training after stroke in the subacute phase (23), recommended a future pairing of questionnaires with interview-based data to better quantify acceptance and explore potential relationships between demographic or functional characteristics and user perspectives.

This mixed-methods study first gathers and explores qualitative data relating to end-user perspectives of using an exoskeleton gait training device in the acute hospital sector after stroke. Thus contributing to the current knowledge gap around usability and acceptance of robotic-assisted gait training in the earlier 
phase of stroke and during a window of potentially enhanced neuroplasticity. In addition, it gathers preliminary quantitative data from Likert scales rating how comfortable and how natural walking feels in the device using different assistance modes and examines the relationship between these scales and participants' ambulatory capacity and stroke related disability.

\section{Materials And Methods}

\section{Ethical Approval}

This study was approved by the hospital's (MMUH) Research Ethics Committee reference 1/378/2052 and written informed consent was obtained from all participants. All methods were performed in accordance with relevant guidelines and regulations.

\section{Participant Recruitment}

Participants were volunteers by self-selection, recruited from an acute hospital setting. Participants were informed of the study by their treating therapist and an information leaflet was provided.

Inclusion criteria required participants to have had a recent hospital admission with stroke, be over 18 years of age, and be medically stable at the time of the research participation. Participants had to measure between 1.58 and $1.88 \mathrm{~m}$ in height with a maximum hip width of $0.46 \mathrm{~m}$ and a maximum weight of $100 \mathrm{~kg}$, to satisfy the dimensions of the exoskeleton. Exclusion criteria included significant cognitive impairments, other neurological conditions or co-existing conditions that limited device use, severe spasticity (modified Ashworth Scale $=4)(24)$, and acute medical illness.

\section{Data Collection}

Following informed consent, participants attended one familiarisation day, where anthropometric measurements were taken and the exoskeleton (EKSO GT, California USA) dimensions were adjusted accordingly for individual participants. Participants completed an introductory RAGT session (lasting approximately 45 minutes). Here step length, step height, and lateral weight transfer targets were individually customised to each participant by a physiotherapist certified in the use of the device. Participants completed repeated $10 \mathrm{~m}$ walks, with rests allowed as required.

At the next training session, participants completed gait training using both maximum assistance (MA) and adaptive assistance (AA) modes. The order of use of each mode was determined by a randomised schedule prepared in advance. In both modes, the exoskeleton was programmed to the "ProStep" mode, in which forward and lateral movement of the users' bodyweight triggers the next step (25). In MA mode the initiation of movement requires only the ability to maintain balance and transfer bodyweight and the device moves the lower limb in the predefined stepping pattern. In AA mode, the participant provides the limb movement where possible, and the device completes the stepping activity where it fails to meet the predefined trajectory parameters. 
Interviews were conducted after the robotic gait training sessions, using a structured but flexible question schedule to allow participants to elaborate freely on their own experiences and perceptions. Key questions asked were: What was your first impression of the device? What did you like about using the device, if anything? What, if anything, do you think using the device could help with during your recovery? What advice would you give to other people who have had a stroke, if they were going to use the device? What ideas do you have for improving the device? What advice would you give to physiotherapists using the device to help make it a good experience for patients?

The semi-structured interviews were audio-recorded, transcribed verbatim and the recordings were subsequently deleted.

Following each training session in maximum and adaptive modes, participants were asked to provide ratings for two questions using 10-point Likert scales. Firstly, 'rate how comfortable you felt during that walk', with 1 being very uncomfortable and 10 being very comfortable. Secondly, 'rate how natural that walk felt to you', with 1 being not natural at all and 10 being very natural. Ratings were recorded for both MA and AA walking modes.

\section{Data analysis}

Content analysis was employed to analyse qualitative interview data and results were synthesised by reporting common meaning units identified in data. Coding was completed by labelling data segments keeping to the participants' own wording as much as possible. As data analysis progressed, data were assembled into categories by identifying common issues, drawing comparisons between participants as well as comparing data from the same participants. To ensure the reliability of the data analysis, two members of the research team (OL) (CMD) reviewed the data and developed the main categories by consensus. This method was deemed suitable as we were conducting exploratory work in an area where not much is known (26).

Summary statistics were used to describe the characteristics of the study participants and to quantify how comfortable and how natural walking in a robotic-assisted gait device felt during the acute and subacute phases of stroke, as rated using the 10-point Likert scales. Spearmann's correlation coefficient explored the relationship between walking capability, stroke related disability and perceptions of device comfort and how natural walking felt. As guided by Cohen (1988, pp 79-81) a rho of 0.1 to 0.29 was considered indicative of a small relationship, 0.3 to 0.49 a medium relationship, and 0.5 to 1.0 as a large relationship between variables (27).

\section{Results}

Ten clinically stable individuals (6 men; 4 women) were recruited in an acute hospital setting (Mater Misericordiae University Hospital, Dublin) following admission with a stroke diagnosis. All ten underwent RAGT following familiarisation, eight of whom consented to the semi-structured interview and nine of whom provided data using the Likert rating scales. 
Participants had a mean age of $64.5 . \pm 12.99$ years and were on average 38.9 days (range 14-79) poststroke when recruited to the study. Eight participants had strokes of ischemic origin, one of haemorrhagic origin, and one with mixed aetiology. All were first episode strokes. One participant, who was not a native English speaker, required a translator for the interview to be conducted and the Likert scales were verbally translated. One participant had expressive aphasia and a closed question interview style was adopted to aid communication during the qualitative interview.

Eight participants (80\%) had post-stroke Functional Ambulation Category (FAC) scores from 0-2 indicating they could not ambulate independently, the remainder $(\mathrm{N}=2)$ could mobilise without physical assistance but required close supervision due to balance problems (28). Participant demographics are summarised in table 1.

Table 1: Participant Demographics

\begin{tabular}{ll}
\hline Participant Demographics & $\mathrm{N}=10$ \\
\hline & $\underline{\text { Mean }(\underline{\mathrm{SD}}) .}$ \\
\hline Age at time of data collection (years) & $64.5 \pm 12.99$ \\
\hline Time since stroke (days) & $35 \pm 21.9$ \\
\hline MRS pre stroke & $0.6 \pm 1.26$ \\
\hline MRS post stroke & $4.1 \pm 0.99$ \\
\hline FAC pre stroke & $5 \pm 0$ \\
\hline FAC post stroke & $1.44 \pm 1.19$ \\
\hline$-\quad \underline{N}(\%)$. \\
\hline Male Gender & $6(60 \%)$ \\
\hline Stroke Laterality- Left & $6(60 \%)$ \\
\hline Stroke type- Ischemic & $9(90 \%)$ \\
\hline
\end{tabular}

\section{Qualitative Interviews}

Content analysis of the interview transcriptions identified primary categories of discussion items that centred around positive aspects of the device, negative aspects, initial concerns relating to the device, the correct mindset for using the device and advice for therapists using the device in an acute rehabilitation setting. Interview data, categories and codes can be found in table 2 .

Table 2: Interview Data 


\begin{tabular}{|c|c|c|c|}
\hline Categories & $\begin{array}{l}\text { Common issues } \\
\text { reported in data } \\
\text { (Codes) }\end{array}$ & $\begin{array}{l}\text { Frequency } \\
\text { of codes* }\end{array}$ & Example of quotes \\
\hline \multirow[t]{6}{*}{$\begin{array}{l}\text { Positive aspects } \\
\text { of robotic gait } \\
\text { device }\end{array}$} & $\begin{array}{l}\text { Belief device is a } \\
\text { beneficial } \\
\text { addition to } \\
\text { rehabilitation }\end{array}$ & 8 & $\begin{array}{l}\text { "Deep down I do say it will do something } \\
\text { good." } \\
\text { "It will probably help me." }\end{array}$ \\
\hline & \multirow[t]{2}{*}{$\begin{array}{l}\text { Intimidating } \\
\text { initially but } \\
\text { subsequently } \\
\text { positive }\end{array}$} & 3 & $\begin{array}{l}\text { "It's quite intimidating looking... 'cause you } \\
\text { don't think you're going to be able to move in } \\
\text { it...And you realize that what the weight is, it's } \\
\text { not as bad as. } \\
\text { "It was crazy!... I enjoyed it" }\end{array}$ \\
\hline & & \multicolumn{2}{|l|}{1} \\
\hline & $\begin{array}{l}\text { Fear of falling in } \\
\text { familiarisation } \\
\text { phase }\end{array}$ & & $\begin{array}{l}\text { "Feelings of fall, you fall, fall on the floor, you } \\
\text { know. In the beginning" }\end{array}$ \\
\hline & \multirow{2}{*}{$\begin{array}{l}\text { Feeling } \\
\text { comfortable } \\
\text { using the device }\end{array}$} & 4 & $\begin{array}{l}\text { "I had no fear of it, even though they were } \\
\text { talking about this that and the other, but I didn't } \\
\text { feel any fear." }\end{array}$ \\
\hline & & & "None of it was ever a bad experience" \\
\hline \multirow[t]{6}{*}{$\begin{array}{l}\text { Negative } \\
\text { aspects of } \\
\text { robotic gait } \\
\text { device }\end{array}$} & $\begin{array}{l}\text { Device fitting } \\
\text { time }\end{array}$ & 2 & $\begin{array}{l}\text { "Too many sensors... too time to, to, yeah, to } \\
\text { put the device on your body. And bells and } \\
\text { stirrups and, yeah, require more shorter time" }\end{array}$ \\
\hline & & \multirow{2}{*}{3} & $\begin{array}{l}\text { "Just the weight... 'cause you really feel like } \\
\text { you're-you're really are putting the effort in" }\end{array}$ \\
\hline & Weight of device & & "The weight was so heavy." \\
\hline & $\begin{array}{l}\text { Degree of verbal } \\
\text { instruction } \\
\text { required from } \\
\text { physiotherapists }\end{array}$ & 1 & $\begin{array}{l}\text { "... I think sometimes the instruction's hard to } \\
\text { understand. Like l'm saying move to the right, } \\
\text { move to the left, that kind of thing sometimes } \\
\text { hard. Maybe less instruction." }\end{array}$ \\
\hline & & 1 & \\
\hline & Dis-comfort & & \\
\hline
\end{tabular}


Advice for future patients using

the device
Have an open mind

1

"Go in with an open mind."

"Not to be afraid of it."

Not to be afraid

Trust the device

"Just go with it. Trusting. They're not going to fall over. The device will hold you up."

2

Relax

Use non-clinical

language

Provide less

instruction

1

Experience using

device

personally

Continue as

currently
"Not to be in a rush like I was... Take it easy."

"Try and relax."

“Explain in layman's terms."

"Maybe less instruction."

"For them to use it first.... To know what it's like. To feel how the patient will feel."

"I don't think there's anything better they can do."

"You couldn't have done anything different."

"They're doing everything they can."

*Note frequency of codes indicates the number of times the code was mentioned in the transcripts.

\section{Positive aspects of robotic gait in acute stroke rehabilitation}

The majority of participants discussed their engagement with the exoskeleton gait device as a positive experience in their rehabilitation. These participants described the device as increasing their confidence and improving their gait.

"It's a good beginning" (Participant \#04)

"It helps you concentrate more on your steps. It's very beneficial. It gives you confidence." (Participant \#10)

"It's just that-that it was doing the job and I was happy with it. It was doing the job right for me." (Participant \#09) 
"It was crazy! Because I didn't know what I'd signed up for. But then I was glad, you know...Because I enjoyed it. None of it was ever a bad experience. Oh, I thought it was great. Couldn't believe it.... that I was walking." (Participant \#01)

The utility of the device during acute stroke rehabilitation for gait restoration was identified.

"I think it's very good because I wasn't able to move around. All I wanted to do was stay in the bed at the hospital. You know? And now I made steps with the help." (Participant \#01)

"Deep down I do say it will do something good." (Participant \#09)

There was one exception where a person who could mobilise independently but with balance deficits did not see a benefit in using the device. He commented:

"I don't think I'd need to [use to help in stroke recovery]" (Participant \#08)

\section{Negative aspects identified during exoskeleton sessions}

A number of participants commented on the time to take measurements, fit and set up the device before initial use. There were approximately 30 minutes total set-up time required for each participant.

"Because you spend, many more time on preparing than going go walking." (Participant \#04)

The weight of the device was considered a negative feature by a number of participants. Some participants commented:

"(tiring) Just the weight.... 'cause you really feel like you're-you're really are putting the effort in" (Participant \#10).

"The weight was so heavy. I find it quite uncomfortable." (Participant \#08)

The degree of verbal instructions provided by physiotherapists during robotic gaits was also discussed:

"... I think sometimes the instructions [are] hard to understand. Like I'm saying move to the right, move to the left, that kind of thing sometimes [was] hard. Maybe less instruction." (Participant \#08)

\section{Initial concerns}

Despite considering the experience positive in general, a number of participants identified that it was intimidating in the initial stages. Participants described worries that the device would be too heavy to mobilise in or that it might restrict their movement.

"It's quite intimidating looking. 'Cause you don't think you're going to be able to move in it. Because it's the big steel frame around you. Yeah, but once you have started... And you realize that... what the weight is, 
it's not as bad as you think it.... you think it's going to restrict your movements also. But it doesn't." (Participant \#10)

[through a translator] He said that it's getting better [from first impression], he said much better. He said very well. [he liked the device?] Yes, decidedly (Participant \#05)

For some, the device was associated with a fear of falling in the familiarisation phase. One participant reported:

"Good device, but very, feelings of fall, [that you might] fall, fall on the floor, you know. In the beginning." (Participant \#04)

Initial feelings of fear were not universal among all participants. One participant reported:

"I had no fear of it, even though they were talking about this that and the other, but I didn't feel any fear." (Participant \#09)

\section{Mindset for people with stroke engaging in exoskeleton training}

Participants identified that nervousness and fear could be an issue or barrier to engagement with the exoskeleton gait training device. When asked what advice they would give future stroke survivors using the device two participants stated:

"Don't be as nervous as myself." (Participant \#02)

"Not to be afraid of it. Not to be in a rush like I was. Take it easy." (Participant \#01)

Participants also advised others to have an open mind when engaging in new or potentially daunting technology:

"Go in with an open mind. Don't be afraid of it because there might be a miracle at the end of it somewhere. You know, again something might click that, it didn't, it might not happen with me, but it could happen with them." (Participant \#09)

Participants identified that there was a need to trust the device, particularly with respect to balance and safety:

"Just go with it. Trusting. They're not going to fall over. The device will hold you up." (Participant \#10)

The need to be relaxed in the device was identified and it was established that when more relaxed the resultant walking was better. One participant advised:

"Try and relax. [Did you find it helpful when you relaxed?] Yeah." (Participant \#08)

\section{Advice for health professions providing exoskeleton training}


Participants identified that it would be beneficial for users of the device to have a clear idea of what was going to happen at every stage of use, and that advice should be given in concise, accessible, and nonclinical language.

"It could be explained to people in layman's terms.... What it would do" (Participant \#09)

One participant stated physiotherapists should personally experience using the device which they felt would help the physiotherapist better understand the experience of patients:

"For them (physiotherapists) to use it first. To get into it and get the-get the-get the swing of it. To know what it's like. To feel how the patient will feel." (Participant \#10)

Overall participants felt the device was a useful addition to rehabilitation. One participant's advice to physiotherapists was:

"Not to stop [using the device]"(Participant \#05)

\section{Likert scale results}

Summary results of ratings of comfort when using the device and how natural walking in the device felt for each exoskeleton mode are depicted in table 3. Overall ratings of device comfort (mean rating 7.95; sd 1.4) and how natural walking felt (mean rating 7.05; sd 1.9) were favourable, with only one participant giving a rating below 5 on any scale (the natural feel of the device). This participant was able to ambulate independently with supervision at the time of data collection. Results indicate that participants rated walking in the device as more comfortable when walking in MA mode (mean Likert score 8.4) when compared to AA mode (mean Likert score 7.6). However, overall participants rated their gait as more natural when in AA mode (mean Likert score 7.3) when compared to MA mode (mean Likert score 6.8).

Table 3: Likert scale results

\begin{tabular}{lll} 
Question & Mode & Mean \pm SD \\
\hline How Comfortable? & Maximum Assist & $8.38 \pm 1.06$ \\
\hline How Comfortable? & Adaptive Assist & $7.56 \pm 1.59$ \\
\hline How Natural? & Maximum Assist & $6.75 \pm 1.75$ \\
\hline How Natural? & Adaptive Assist & $7.33 \pm 2.18$
\end{tabular}

Note: Likert scale where $1=$ very uncomfortable and $10=$ very comfortable or $1=$ not natural at all and 10 = very natural

The relationship between both participants' stroke-related ambulatory category as measured by the FAC and disability as measured by the mRS and the Likert rating scales were next explored using Spearmann's correlation coefficient. Of note, a strong and positive relationship (rho=0.6) between the mRS and how 
natural walking in the device was rated in MA mode was identified, whereby the higher the level of disability, the more natural participants rated walking in the device in MA mode. A moderate and negative relationship was observed between FAC and how natural walking in the device was rated in MA, again meaning those more independent in ambulation (higher FAC), rated walking in in MA mode as less natural. Overall these trends point to more dependent individuals rating walking in the device as more natural. In contrast, a moderate positive relationship was found between FAC and comfort in AA mode, with a corresponding negative relationship for MRS, pointing to more physically independent and ambulatory participants rating the device as more comfortable when they could contribute to the movement.

Results from Spearman's correlation coefficient of the relationship between ambulatory ability (FAC) and disability (mRS) and Likert scales in AA and MA mode can be found in table 4.

Table 4: Spearman's correlation coefficient results

\section{Comfort MA Comfort AA Natural MA Natural $A A$}

Disability scale

\begin{tabular}{lllll} 
& rho & rho & rho & rho \\
\hline$F A C$ & 0.09 & 0.36 & -0.33 & -0.18 \\
\hline$m R S$ & 0.06 & -0.3 & 0.62 & 0.05
\end{tabular}

MA: maximum assistance; AA: Adapt assist; FAC: functional ambulatory category; mRS: modified Rankin scale for disability

\section{Discussion}

This study provided new insights into robotic-assisted gait devices when utilised in the acute hospital setting from individuals in the acute and subacute phases of stroke. The results of this study suggest individuals in the early phases of stroke perceive a robotic-assisted gait device to be acceptable and helpful in the main. Participants identified set-up time, weight of the device, and requirement of multiple instructions by therapists during walking as negative aspects of the device. The majority of participants believed that the device could provide gait training benefits and would choose to continue using the device. On average the device was rated as comfortable to use and the robotic-assisted walking in both maximum and adaptive assistive modes were rated as natural.

Qualitative meta-synthesis of robotics in rehabilitation identifies that robotic therapy is perceived as enjoyable, but also tiring, frustrating and difficult (20). These sentiments were mirrored to a large degree in our research in the acute hospital setting after stroke where participants were positive about their experiences of RAGT but with caveats that related to the weight and restrictive nature of the device and the need for repeated therapist verbal instructions during use. Despite these, the majority of participants 
in the current study considered RAGT as useful and beneficial and would choose to add RAGT to their rehabilitation programme, if given the choice. In stroke, comparative qualitative data relating to RAGT are limited and conflicting. A study by Vaughan-Graham et al. (2020) reported that chronic stroke survivors while hopeful about exoskeleton technology, expressed disappointment that the technology did not yet meet their expectations (22). In contrast a recent study conducted in a subacute stroke population reported positive perceptions of exoskeleton-based physiotherapy which better echoing the findings in this study, where patients rated it favourably, attributing opportunity and benefit to using the device during subacute rehabilitation (23).

Time demands for device set-up was one limitation highlighted in the current study that may potentially impede widespread uptake among acute and subacute stroke survivors within a clinical facility. The amount of time required to record individual anthropometric measurements and fit the device were identified as negative aspects. This limitation was mirrored in other qualitative studies examining device users' $(21,22,23)$ and therapists' perspectives $(23,29,30,31)$ of RAGT. However, this current study recorded only early perceptions following each participant's first two trials of using the device where the burden of assessment and setup has previously been reported as highest (30). Similarly, while individuals in the current study identified the technology was daunting at first, studies with longer training periods have identified a phase described by patients as "getting into the swing of things" (32). Qualitative data addressing both user and therapists' perceptions of the device previously highlighted the importance of committing to a minimum number of training sessions when integrating an exoskeleton into stroke rehabilitation, and that perseverance through early difficulties is necessary to attain efficient exoskeleton training (24). This finding is further supported by a feasibility study that examined the integration of RAGT in inpatient rehabilitation, where therapists initially reported that time limitations reduced the effectiveness of the device but reported an improvement in feasibility over time (29).

This study adds to the knowledge base of patient experiences of using RAGT following stroke by providing quantitative ratings relating to walking in a robotic device. Data generated some interesting findings. While all participants rated the device as relatively comfortable ( $>5$ on the Likert scale), the MA mode (where the person is a passive passenger in the device) was perceived to be more comfortable than the AA mode (where the person contributes to the movement). One reason participants may have perceived MA mode to be more comfortable is that they are not attempting a movement that may be at odds with the programmed trajectory. RAGT in healthy individuals has previously been noted to limit lower limb and pelvic degrees of freedom resulting in altered muscle activation patterns (32). A second reason for this finding may be as a result of the increased energy demands of walking in AA mode when compared to MA mode. While there is no comparable evidence published in stroke, a study in incomplete spinal cord injury found a wide variation in cardiorespiratory and metabolic responses based on the modes of assistance used, with the maximum assist mode reported to be the least demanding on cardiorespiratory and metabolic functions (33).

All participants in this study again gave a relatively positive rating for how natural walking in the device felt ( $>5$ on the Likert scale). Here, in contrast to the findings in relation to comfort, ratings were higher in 
the AA mode, where the individual contributes to the stepping trajectory. We hypothesise that it feels more natural for participants to initiate walking themselves and have more autonomy over the movement than to be a passive passenger in the device. While no data were identified in the published literature for direct comparison between robotic modes of assistance, a previous study reported individuals with spinal cord injury reported walking felt more natural when they had the autonomy to trigger a step forward using forward and lateral weight shift targets in comparison to when it was triggered by a therapist, even if they were not always in tune with the device (34). It is interesting to note that in the current study that a strong linear relationship was found between disability level (measured by the mRS) and the natural feel of the device in MA mode, where a higher level of disability related to a more natural perception of walking in the device in MA mode. This supports the interpretation that an individual's perception of how natural robotic walking feels is dependent on their level of impairment and their current compensatory techniques during normal overground walking. Current robotic exoskeleton devices may promote more unnatural, non-physiological walking characteristics to those of an independent ambulator's natural gait. Evidence supports that walking in a robotic gait device (such as EKSO) is dissimilar to normal overground walking with regard to lower extremity muscle activity and joint motions. In healthy individuals this manifests as slower walking, with shorter steps and greater double-limb support time when using the device $(35,36)$. However, EMG and EEG studies in robotic-assisted gait identify greater movement intent and muscle contribution in active rather than passive walking in robotic-assisted gait devices (37) and it is this active contribution to gait that may be driving the higher ratings for natural gait in active assistance mode.

\section{Conclusion}

Overall exoskeleton gait training was deemed to be an acceptable intervention that was comfortable, natural and helpful in gait rehabilitation for individuals with stroke in the acute hospital setting.

Participants identified that the technology could be daunting at first and that people using the device should keep an open mind, relax, and trust the device and therapists. Some negative aspects identified included device set-up time, the weight of the device, and the need to respond to multiple verbal commands when walking in the device.

\section{List Of Abbreviations}

RAGT= Robotic Assisted Gait Training

MA mode $=$ Maximum Assistance Mode

AA mode $=$ Adaptive Assistance Mode

$\mathrm{FAC}=$ Functional Ambulation Category

mRS = Modified Rankin Scale

\section{Declarations}




\section{Ethics approval and consent to participate}

This study was approved by the hospital's (MMUH) Research Ethics Committee reference 1/378/2052 and written informed consent was obtained from all participants.

\section{Consent for publication}

Written informed consent was obtained from all participants.

\section{Availability of data and materials}

The data that support the findings of this study are not openly available due to reasons of sensitivity and are available from the corresponding author upon reasonable request.

\section{Competing interests}

The authors declare that they have no competing interests.

\section{Funding}

This study was supported by EU H2020 MSCA RISE grant: PRO GAIT reference 778043.

\section{Authors' contributions}

OL and CF performed data collection. OL and CMD analysed and interpreted the collected data and contributed to writing the manuscript. All authors read, edited and approved the final manuscript.

\section{Acknowledgements}

Not Applicable

\section{References}

1. Global Burden of Disease Collaborative Network. Global Burden of Disease Study 2019 (GBD 2019) Results [Internet]. Seattle (WA): Institute for Health Metrics and Evaluation. Available at: http://ghdx.healthdata.org/gbd-results-tool

2. Belda-Lois JM, Mena-del Horno S, Bermejo-Bosch I, Moreno JC, Pons JL, Farina D, losa M, Molinari M, Tamburella F, Ramos A, Caria A. Rehabilitation of gait after stroke: a review towards a top-down approach. Journal of neuroengineering and rehabilitation. 2011 Dec;8(1):1-20.

3. Flansbjer UB, Holmbäck AM, Downham D, Patten C, Lexell J. Reliability of gait performance tests in men and women with hemiparesis after stroke. Journal of rehabilitation medicine. $2005 \mathrm{Mar}$ 1;37(2):75-82.

4. Geyh S, Cieza A, Schouten J, Dickson H, Frommelt P, Omar Z, Kostanjsek N, Ring H, Stucki G. ICF Core Sets for stroke. Journal of rehabilitation medicine. 2004 Aug 1;36(0):135 - 41. 
5. Hill K, Ellis P, Bernhardt Jet al. (1997) Balance and mobility outcomes for stroke patients: a comprehensive audit. Aust J Physiother, 43, 173-180.

6. Park J, Lee SU, Jung SH. Prediction of post-stroke functional mobility from the initial assessment of cognitive function. NeuroRehabilitation. 2017 Jan 1;41(1):169 - 77.

7. Bohannon RW, Andrews AW, Smith MB. Rehabilitation goals of patients with hemiplegia. International Journal of Rehabilitation Research. 1988 Jun 1;11(2):181-4.

8. Kramer S, Johnson L, Bernhardt J, Cumming T. Energy expenditure and cost during walking after stroke: a systematic review. Archives of physical medicine and rehabilitation. 2016 Apr 1;97(4):61932.

9. Janet H Carr EdD FACP, Roberta B Shepherd EdD FACP; Stroke Rehabilitation- Guidelines for Exercise and Training to Optimize Motor Skill; First edition; 2003

10. Pollock A, St George B, Fenton M, Firkins L. Top ten research priorities relating to life after stroke. The Lancet Neurology. 2012 Mar 1;11(3):209.

11. French B, Thomas LH, Coupe J, McMahon NE, Connell L, Harrison J, Sutton CJ, Tishkovskaya S, Watkins CL. Repetitive task training for improving functional ability after stroke. Cochrane database of systematic reviews. 2016(11).

12. Veerbeek JM, van Wegen E, van Peppen R, van der Wees PJ, Hendriks E, Rietberg M, Kwakkel G. What is the evidence for physical therapy poststroke? A systematic review and meta-analysis. PloS one. 2014 Feb 4;9(2):e87987.

13. Bruni MF, Melegari C, De Cola MC, Bramanti A, Bramanti P, Calabrò RS. What does best evidence tell us about robotic gait rehabilitation in stroke patients: a systematic review and meta-analysis. Journal of Clinical Neuroscience. 2018 Feb 1;48:11 - 7.

14. Mehrholz J, Thomas S, Kugler J, Pohl M, Elsner B. Electromechanical-assisted training for walking after stroke. Cochrane database of systematic reviews. 2020(10).

15. Calabrò RS, Naro A, Russo M, Bramanti P, Carioti L, Balletta T, Buda A, Manuli A, Filoni S, Bramanti A. Shaping neuroplasticity by using powered exoskeletons in patients with stroke: a randomized clinical trial. Journal of neuroengineering and rehabilitation. 2018 Dec;15(1):1-6.

16. Rehme AK, Eickhoff SB, Rottschy C, Fink GR, Grefkes C. Activation likelihood estimation metaanalysis of motor-related neural activity after stroke. Neuroimage. 2012 Feb 1;59(3):2771-82.

17. Coleman ER, Moudgal R, Lang K, Hyacinth HI, Awosika OO, Kissela BM, Feng W. Early rehabilitation after stroke: a narrative review. Current atherosclerosis reports. 2017 Dec;19(12):1-2.

18. Jørgensen HS, Nakayama H, Raaschou HO, Vive-Larsen J, Støier M, Olsen TS. Outcome and time course of recovery in stroke. Part I: Outcome. The Copenhagen Stroke Study. Archives of physical medicine and rehabilitation. 1995 May 1;76(5):399-405.

19. Kreisel SH, Hennerici MG, Bäzner H. Pathophysiology of stroke rehabilitation: the natural course of clinical recovery, use-dependent plasticity and rehabilitative outcome. Cerebrovascular diseases. 2007;23(4):243-55. 
20. Laparidou D, Curtis F, Akanuwe J, Goher K, Niroshan Siriwardena A, Kucukyilmaz A. Patient, carer, and staff perceptions of robotics in motor rehabilitation: a systematic review and qualitative metasynthesis. Journal of neuroengineering and rehabilitation. 2021 Dec;18(1):1-24.

21. Danzl MM, Chelette KC, Lee K, Lykins D, Sawaki L. Brain stimulation paired with novel locomotor training with robotic gait orthosis in chronic stroke: a feasibility study. NeuroRehabilitation. 2013 Jan 1;33(1):67-76.

22. Vaughan-Graham J, Brooks D, Rose L, Nejat G, Pons J, Patterson K. Exoskeleton use in post-stroke gait rehabilitation: a qualitative study of the perspectives of persons post-stroke and physiotherapists. Journal of neuroengineering and rehabilitation. 2020 Dec;17(1):1-5.

23. Louie DR, Mortenson WB, Lui M, Durocher M, Teasell R, Yao J, Eng JJ. Patients' and therapists' experience and perception of exoskeleton-based physiotherapy during subacute stroke rehabilitation: a qualitative analysis. Disability and Rehabilitation. 2021 Oct 20:1-9.

24. Meseguer-Henarejos AB, Sanchez-Meca J, Lopez-Pina JA, Carles-Hernandez R. Inter-and intra-rater reliability of the Modified Ashworth Scale: a systematic review and meta-analysis. European journal of physical and rehabilitation medicine. 2017 Sep 13;54(4):576-90.

25. Gandolla M, Guanziroli E, D’Angelo A, Cannaviello G, Molteni F, Pedrocchi A. Automatic Setting Procedure for Exoskeleton-Assisted Overground Gait: Proof of Concept on Stroke Population. Front Neurorobot [Internet]. 2018 Mar 19;12(MAR):1-11.

26. Holmberg C. Judith Green \& Nicki Thorogood (2004). Qualitative Methods for Health Research. InForum Qualitative Sozialforschung/Forum: Qualitative Social Research 2006 Mar 31 (Vol. 7, No. 2).

27. Cohen, JW (1988). Statistical power analysis for the behavioural sciences. Hillsdale, NJ: Lawrence Erlbaum Associates. 1988.

28. Mehrholz J, Wagner K, Rutte K, Meißner D, Pohl M. Predictive validity and responsiveness of the functional ambulation category in hemiparetic patients after stroke. Archives of physical medicine and rehabilitation. 2007 Oct 1;88(10):1314-9.

29. Swank C, Sikka S, Driver S, Bennett M, Callender L. Feasibility of integrating robotic exoskeleton gait training in inpatient rehabilitation. Disability and Rehabilitation: Assistive Technology. 2020 May 18;15(4):409 - 17.

30. Read E, Woolsey C, McGibbon CA, O'Connell C. Physiotherapists' experiences using the Ekso bionic exoskeleton with patients in a neurological rehabilitation hospital: A qualitative study. Rehabilitation research and practice. 2020 Jan 8;2020.

31. Mortenson WB, Pysklywec A, Chau L, Prescott M, Townson A. Therapists' experience of training and implementing an exoskeleton in a rehabilitation centre. Disability and Rehabilitation. $2020 \mathrm{Jul}$ 9:1-7.

32. Hidler JM, Wall AE. Alterations in muscle activation patterns during robotic-assisted walking. Clinical Biomechanics. 2005 Feb 1;20(2):184 - 93.

33. Kressler J, Wymer T, Domingo A. Respiratory, cardiovascular and metabolic responses during different modes of overground bionic ambulation in persons with motor-incomplete spinal cord injury: A case series. Journal of rehabilitation medicine. 2018 Feb 5;50(2):173 - 80. 
34. Thomassen GK, Jørgensen V, Normann B. "Back at the same level as everyone else"-user perspectives on walking with an exoskeleton, a qualitative study. Spinal cord series and cases. 2019 Dec 13;5(1):1-7.

35. Swank C, Wang-Price S, Gao F, Almutairi S. Walking with a robotic exoskeleton does not mimic natural gait: a within-subjects study. JMIR rehabilitation and assistive technologies. 2019 Jan 14;6(1):e11023.

36. De Luca A, Bellitto A, Mandraccia S, Marchesi G, Pellegrino L, Coscia M, Leoncini C, Rossi L, Gamba $S$, Massone A, Casadio M. Exoskeleton for gait rehabilitation: effects of assistance, mechanical structure, and walking aids on muscle activations. Applied Sciences. 2019 Jan;9(14):2868.

37. García-Cossio E, Severens M, Nienhuis B, Duysens J, Desain P, Keijsers N, Farquhar J. Decoding sensorimotor rhythms during robotic-assisted treadmill walking for brain computer interface (BCI) applications. PloS one. 2015 Dec 16;10(12):e0137910. 